\title{
A Pilot Study of Enhance Recovery after Surgery (ERAS) for Liver Resection
}

\author{
Jarmin $\mathrm{R}^{\mathrm{a}}$, Mohamad IS ${ }^{\mathrm{b}}$, Ahmad AW', Othman $\mathrm{H}^{\mathrm{a}}$, Zuhdi $\mathrm{Z}^{\mathrm{a}}$, Ariffin $\mathrm{AC}^{\mathrm{d}}$ \\ ${ }^{a}$ Department of Surgery, Universiti Kebangsaan Malaysia \\ ${ }^{b}$ Department of Surgery, Universiti Sains Malaysia \\ ${ }^{c}$ Faculty of Medicine, International Islamic University of Malaysia \\ ${ }^{d}$ Department of Surgery, Universiti Sains Islam Malaysia
}

\begin{abstract}
Background: Morbidity post hepatectomy still remain persistent throughout decades compared to other surgery. Modern approach have been introduced to improve safety and reduce morbidity whilst at the same time enhance patient recovery. Thus, enhanced recovery after surgery or fast track recovery program for liver resection was initiated. Objective: The aim of this study was to achieve discharge by postoperative day 3 for minor resection and day 5 for major resection. Design and Setting: This is a prospective study conducted in Hospital Universiti Kebangsaan Malaysia (HUKM) from September 2014 till April 2015. Material and Methods: All patients undergoing open liver resection were included in the study. They were then managed post operatively according to ERAS protocol that was drawn up based on previous studies. Patient's demographics data, intra operative parameters, postoperative complications and adherence to postoperative recovery protocol were recorded. Results: Seventeen patients (7 major and 10 minor resection) were recruited. The mean length of hospital stay for minor resection was 5.9 and major resection was 9.6. With regards to the targets, 4 out of 10 (40\%)patients in minor resection group and 4 out 7 (57.1\%) in the major group were discharged on time. 9 patients had postoperative complications with no mortality recorded. In terms of the ERAS protocol targets, the PCA morphine discontinuation target was achieved in 15 patients $(88.3 \%)$, nasogastric tube removal (13 patients $-76.5 \%)$, urinary cathether removal (6 patients $35.3 \%$ ), abdominal drains removal ( 9 patients-52.9\%) and resumption of full diet was achieved by $82.4 \%(14$ patients). Conclusion: From these overall achievement, most of our targets have been met and this shows that our ERAS protocol is safe to be applied to patient undergoing hepatectomy. Limitations: Some patients had achieved their target but not discharged for unknown reason.
\end{abstract}

KEYWORDS: hepatectomy; enhanced recovery after surgery; fast track recovery; liver resection

\section{INTRODUCTION}

Enhanced recovery after surgery (ERAS) or also known as fast track recovery program comprises of multidisciplinary approach aiming to reduce surgical stress response, enhance immune function and thereby reduce organ dysfunction and allow for faster recovery after surgery.

The concept of ERAS was first introduced by Kehlet et al. (1997). ${ }^{1}$ The idea came from the surgical procedures that are now safely done in daycare OT such as herniorraphy, laparoscopic cholecystectomy and eye surgery. The principles of short hospital stay and early return to ambulatory condition are being extended to more complex

Corresponding author:

Dr. Ikhwan Sani Mohamad

MD (USM), MMed (SURGERY USM)

Department of Surgery,

Universiti Sains Malaysia,

16150 Kubang Kerian ,

Kota Bharu, Kelantan, Malaysia.

Tel no: +60179278535

Email : ikhwansani@yahoo.com.my elective surgical procedures with minimally invasive procedures and also regional anaesthesia being integral to the program.

The most obvious benefit of such program is to expedite return to normal function whilst reducing hospital stay and cost incurred as well as reducing the associated hospitalised associated complication such as pneumonia, thromboembolism etc. At the same time, this program should also be safe in terms of prevention of complication and morbidity related to the surgery in the perioperative period.

Liver resections has been regarded as high risk surgeries which traditionally involved the use invasive monitoring, long period of surgery, significant blood loss and long hospital stay. With ERAS, multimodal approach is adopted involving surgeons, anaesthesiologist and nurses to apply all the perioperative management of patient undergoing liver resection. The key elements involve optimization of the patient perioperative condition, short acting general anaesthetic agent, limited use of catheters, drains and tubes, opioidsparing analgesia and enforced early mobilization and oral nutrition. 
The aim of the study was to measure the effect of the ERAS protocol on safety and length of hospital stay following liver resection.

\section{METHODS}

We collected data prospectively from patients undergoing hepatectomy at our medical centre from April 2015-October 2015. All patients will be informed to be involved in the study. Exclusion criterion is the involvement of any other secondary procedures such as hepaticojejunostomy and colectomy.

Liver resection are categorized as minor resection (fewer than 3 segment including multiple non anatomical wedge resection) and major resection (3 or more segments).

The incision of choice for surgery is right subcostal incision. All patients are managed intraoperatively with compression stocking and sequential calf compression pump, urinary catheter and arterial line. All patients received DVT prophylaxis. All incisions closed in layers with loop Ethilon 1 (Ethicon) and Fixed Head Skin Staplers (Ethicon) to skin.

Patients received standardized postoperative care according to the ERAS protocol as described in Table 1.

Table 1: Standardized protocol for ERAS in HUKM for this study.

\begin{tabular}{|c|c|c|}
\hline & Minor resection & Major resection \\
\hline \multicolumn{3}{|l|}{ Immediate Post Operation } \\
\hline Ward Type & Ward & Ward/ High Dependency Unit \\
\hline Urinary cathether & Keep & Keep \\
\hline Nasogastric tube & Keep & Keep \\
\hline Drain & Optional & Mandatory \\
\hline Mobility & Bed rest & Bed rest \\
\hline Nutrition & Nil & Nil \\
\hline Analgesia & IV PCA Morphine +/- Parecoxib & IV PCA Morphine +/- Parecoxib \\
\hline \multicolumn{3}{|l|}{ Postoperative Day1 } \\
\hline Ward Type & Ward & Transfer out High Dependency Unit \\
\hline Urinary Cathether & Remove if good urine output & Remove if good output \\
\hline Nasogastric tube removal & Remove if $<100 \mathrm{ml}$ & $<100 \mathrm{ml}$ \\
\hline Drain removal & $<50 \mathrm{ml}$ & $<50 \mathrm{ml}$ \\
\hline Mobility & Sit up on lazy chair & Sit up in bed \\
\hline Nutrition & Allow oral fluids to soft diet & Allow oral fluids \\
\hline Analgesia & IV PCA Morphine +/- Parecoxib & IV PCA Morphine +/- Parecoxib \\
\hline \multicolumn{3}{|l|}{ Post Operative Day2 } \\
\hline Urinary Cathether & Remove & Remove if good urine output \\
\hline Mobility & Mobilise $>8$ hours/day & Sit up in lazy chair \\
\hline Drain removal & $<<50 \mathrm{ml}$ & $<50 \mathrm{ml}$ \\
\hline Nutrition & Full diet & Soft diet \\
\hline Analgesia & $\begin{array}{l}\text { Etoricoxib + Paracetamol or Tra- } \\
\text { madol }\end{array}$ & Etoricoxib + Paracetamol or Tramadol \\
\hline \multicolumn{3}{|l|}{ Post Operative Day3 } \\
\hline Drain removal & Remove & Remove if $<50 \mathrm{ml}$ \\
\hline Mobility & Full mobility & Mobilise $>8$ hours/day \\
\hline Nutrition & Full diet & Full diet \\
\hline Analgesia & Etoricoxib + Parcetamol or Tramadol & Etoricoxib + Paracetamol or Tramadol \\
\hline \multirow[t]{2}{*}{ Investigation } & Liver Function Test & Liver Function Test \\
\hline & Consider discharge & \\
\hline \multicolumn{3}{|l|}{ Post Operative Day4 } \\
\hline Drain removal & - & Remove if $<50 \mathrm{ml}$ \\
\hline Mobility & - & Full mobility \\
\hline Nutrition & - & Full diet \\
\hline \multicolumn{3}{|l|}{ Post Operative Day5 } \\
\hline investigation & - & Liver Function Test \\
\hline Discharge & - & Aim discharge if met 50:50 criteria \\
\hline
\end{tabular}




\section{STATISTICAL ANALYSIS}

Continuous data are expressed as mean or median (range). The Mann Whitney U Test was used to find association between intraoperative event and length of stay. Data were analyzed using SSPS version 19.0 for Windows. $P<0.05$ was considered significant.

\section{RESULTS}

The study recruited 17 patients within the study period (10 minor resection, 7 major resection). The mean age of patients in this study was 54.6 years (range 24-76). There was no difference in demographics between both groups. (Figure 1)

The median operating time was 275 minutes. The median blood loss was $675 \mathrm{ml}$ for minor group and $1000 \mathrm{ml}$ for major group.

No mortality was reported. 5 patients in minor group and 4 patients in the major group had postoperative complications. Based on Clavien Dindo classification only $6 \%$ patient is classified as 3 and none in class 4 or 5 (Figure 2$)^{2}$

\begin{tabular}{|c|c|c|c|}
\hline & $\begin{array}{l}\text { All } \\
\text { patients } \\
(\mathrm{n}=17)\end{array}$ & $\begin{array}{l}\text { Minor } \\
\text { resection } \\
(\mathrm{n}=10)\end{array}$ & $\begin{array}{l}\text { Major } \\
\text { resection } \\
(\mathrm{n}=7)\end{array}$ \\
\hline Age & 54.6 & 58.1 & 51.1 \\
\hline \multicolumn{4}{|l|}{ Gender } \\
\hline Male & 10 & 6 & 4 \\
\hline Female & 7 & 4 & 3 \\
\hline \multicolumn{4}{|l|}{ Diagnosis } \\
\hline $\begin{array}{l}\text { Benign mucinous } \\
\text { cystadenoma }\end{array}$ & 1 & 1 & 0 \\
\hline Cholangiocarcinoma & 1 & 0 & 1 \\
\hline $\begin{array}{l}\text { Colorectal liver } \\
\text { mets }\end{array}$ & 6 & 1 & 5 \\
\hline $\begin{array}{l}\text { Gallbladder } \\
\text { carcinoma }\end{array}$ & 1 & 1 & 0 \\
\hline $\begin{array}{l}\text { Hepatocellular } \\
\text { Carcinoma }\end{array}$ & 6 & 5 & 1 \\
\hline Hepatolithiasis & 1 & 1 & 0 \\
\hline $\begin{array}{l}\text { Recurrrent complex } \\
\text { liver cystadenoma } \\
\text { with metastatic } \\
\text { malformation }\end{array}$ & 1 & 1 & 0 \\
\hline $\begin{array}{l}\text { Operative } \\
\text { parameters } \\
\text { Duration of } \\
\text { Operation }(\mathrm{min})\end{array}$ & 275 & 219 & 420 \\
\hline Blood loss (ml) & 1000 & 675 & 1000 \\
\hline
\end{tabular}

Figure 1: Patients Demographic data

\begin{tabular}{lll}
$\begin{array}{l}\text { Clavien Dindo } \\
\text { classification }\end{array}$ & number & $\%$ \\
\hline class 1 & 4 & $23.52 \%$ \\
class 2 & 4 & $23.52 \%$ \\
class 3 & 1 & $5.88 \%$ \\
class 4 & 0 & $0.00 \%$ \\
class 5 & 0 & $0.00 \%$ \\
No post op complication & 8 & $47.05 \%$
\end{tabular}

Figure 2 : Clavien Dindo classification of postoperative complications

\section{ERAS performance}

The mean discharge day was 5.9 days (range 3-8 days) for minor resection and 9.6 days (range 5-19 days) for major resection. 8 patients $(47.1 \%)$ were discharged on the set date ( 4 for each group).

PCA (patient controlled analgesia) infusion of morphine $1 \mathrm{mg} / \mathrm{ml} /$ dose was used for most patients (82.35\%), followed by epidural (11.76\%) and SC (subcutaneous injection) morphine $(5.88 \%)$. The mean duration of PCA morphine use was 3 days. Overall by POD 3,15 patients $(88.3 \%)$ have established oral analgesia.

The target for nasogastric tube removal was Day 1 for minor group and Day2 for major group. For minor resection, 5 patients had nasogastric tube removed on immediately post op and 3 patients on Day 1. For major resection, 2 patients had nasogastric tube removed the same day and 3 patients on Day1. Target for removal was achieved by $76.5 \%$.

The decision for removal of urinary catheter was based on the urine output (at least $0.5 \mathrm{ml} / \mathrm{kg} / \mathrm{hr}$ ). The target removal of urinary catheter was set at POD 2 for minor resection and POD3 for major resection. Target for cathether removal on the set date achieved in $35.3 \%$.

The mean duration of drain use was 4 days (range 0 17 days). The target for drain removal was set at Day3 (minor) and Day5 (major). Mean time of removal for each group was 3 days (range 0-6 days) for minor and 7 days (range 1-17 days) for major resection. Target for drainage removal was achieved in $52.9 \%$.

The resumption of the ability to take full diet was one of the discharge criteria. The target was set on Day 3 for minor resection and Day 4 for major resection. The mean for minor resection was 3 days (range 1-4 days). The mean for major resection was 4 days (range $2-7$ days). $82.4 \%$ patient resume to full diet on the desired date. 


\begin{tabular}{llll}
\hline Parameters & \multicolumn{3}{l}{ Achieved Desired Duration (n) } \\
\cline { 2 - 4 } & $\begin{array}{l}\text { All patients } \\
(\mathrm{n}=17)\end{array}$ & $\begin{array}{l}\text { Minor } \\
\text { resection } \\
(\mathrm{n}=10)\end{array}$ & $\begin{array}{l}\text { Major } \\
\text { Resection } \\
(\mathrm{n}=7)\end{array}$ \\
\hline $\begin{array}{l}\text { Non oral } \\
\text { analgesia } \\
\text { use }\end{array}$ & $15(88.3 \%)$ & $10(100 \%)$ & $5(71.4 \%)$ \\
$\begin{array}{l}\text { Duration of } \\
\text { Nasogastric }\end{array}$ & $13(76.5 \%)$ & $8(80 \%)$ & $5(71.4 \%)$ \\
$\begin{array}{l}\text { Tube } \\
\text { Duration of } \\
\text { urinary } \\
\text { cathether } \\
\text { Duration of } \\
\text { drainage }\end{array}$ & $6(35.3 \%)$ & $3(30 \%)$ & $3(42.9 \%)$ \\
$\begin{array}{l}\text { Initiation of } \\
\text { diet } \\
\text { Length of }\end{array}$ & $14(82.4 \%)$ & $9(90 \%)$ & $5(71.4 \%)$ \\
$\begin{array}{l}\text { Stay } \\
\text { (LOS) }\end{array}$ & $8(47.1 \%)$ & $4(40 \%)$ & $4(57.1 \%)$ \\
\hline
\end{tabular}

Figure 3 : Key Target Performance Criteria

\section{DISCUSSION}

Enhanced recovery after surgery (ERAS) programme has been introduced since the early 1997 by Kehlet et. al. ${ }^{1}$ The concept has been well researched and documented especially in colorectal cancer. ${ }^{3}$ Metaanalysis by Varadhan et al. (2010) have showed that ERAS in open colorectal surgery have reduced length of hospital stay and complication rates compared to conventional method. ${ }^{4}$

Based on these evidences, hepatobiliary surgeons have started to adopt ERAS concept in order to improve their outcome. Previous studies have showed that ERAS in hepatectomy is feasible and associated with reduced length of hospital stay (LOS). Previously published data have quoted as early as POD 4 in minor resection (Mackay \& O'Dwyer. ${ }^{5}$ Van Dam et al. reported LOS of 6 days following open hepatectomy]. ${ }^{6}$ Another study by Capusotti et al. found a mean LOS of 8.8 days. $^{7}$

Our target discharge day for minor resection was POD 3 and major resection was POD 5. We were able to discharge 8 patients $(47.1 \%)$ on the set day. With regards to the minor resection, we have achieved $5.9+/-1.73$ SD (range 3-8 days) which is 2 days longer than the quoted target but we have achieved discharge on day 3 post minor resection without any increase in morbidity, mortality or readmission. For major resection, the results were $9.6+/-5.2$ SD (range 5-19 days). The results were skewed due to the presence of 2 cases that required long stay (POD 13 and POD 19).

A previous study by Jarnagin et al. (2002) showed that the number of hepatic segment and estimated blood loss serves as an independent predictors of both perioperative morbidity and mortality. ${ }^{8}$ Interestingly, in our study we have no found any statistically significant correlation between estimated blood loss, hepatic segment resected or duration of the operation. But, admittedly our data set was small thus further number required to reach a statistically significant conclusion.

Previous study have recorded that liver surgery is associated with morbidity of $38-45 \%$ and mortality of $2.7-3.1 \%{ }^{9,10}$ We recorded complication in 9 patients $(52.9 \%)$. The complications were hospital acquired pneumonia, ascites, pleural effusion, coagulopathy, surgical site infection, ileus, acute kidney injury, liver failure and bleeding. There were no mortality recorded throughout the study. Based on Clavien Dindo classification none of the patient is in Class 4 or $5 .^{2}$

Administration of adequate analgesia play an important role in patient's postoperative recovery. ERAS concept suggest the usage of opioid sparing regime to prevent reduce gastric stasis and also allow for early ambulation. Meta-analysis (Block et al. 2003) have shown that epidural has better postoperative pain control compared to parenteral opioid in open abdominal surgery. ${ }^{11}$ For this study, 15 patients had PCA morphine infusion, whilst 2 had epidural infusion and 1 patient had SC morphine injection. Our aim was to discontinue the usage of these analgesic modalities and establish oral analgesia as early as POD 2. However, we allow for violation of protocol based patient clinical need that is assessed by the pain control team. From our observation, almost all patients $(15 / 17=88.3 \%)$ had been converted to oral analgesia on Day 3 .

Nasogastric tube was placed during the surgery to aid decompression of the stomach to have better exposure during surgery. Early removal of nasogastric tube postoperatively was reported by Nelson et.al in a cochrane study to give early return of bowel function, reduce pulmonary complication and reduced length of stay. ${ }^{12} 13$ patients $(76.5 \%)$ achieved target of nasogastric tube removal of which 7 patients $(41.2 \%)$ had the tube removed immediately postoperative. The other 2 cases were removed on Day 3 and Day 4 due to prolonged intubation and ileus.

Early removal of the urinary catheter will reduce the risk of developing urinary tract infection and expedite early ambulation. We set the criteria target day for removal of urinary catheter was based on the urine output at least $0.5 \mathrm{ml} / \mathrm{kg} / \mathrm{hr}$. The target removal of urinary catheter was set at POD 2 for minor resection and POD3 for major resection. Only 6 patients $(35.3 \%)$ had their urinary catheter removed on the target day. The presence of urinary catheter will certainly delay ambulation and subsequently increase length of stay.

The usage of abdominal drains will help detection of major complications such as bleeding or bile leak and acts a control fistula to prevent serious sepsis from developing unnoticed. However, studies have shown that routine usage of drain after hepatectomy is not related or predictive of 
postoperative complications. ${ }^{13}$ Based on other ERAS protocol, we advocate optional usage of drain for minor resection but mandatory for major resection. The target for drain removal was set at POD 3 (minor) and POD 5 (major). With these targets, 3 patients $(17.6 \%)$ in the minor group did not have drains. 9 patients $(52.97 \%)$ had achieved the designated target of the ERAS protocol. In the major group, the patients have delayed removal of drains due to bilious drainage, high output and also coagulopathy.

The next important target and part of discharge criteria is resumption of full oral feeding. It is important to achieve this target as it will be important for patient to maintain adequate nutrition at home to promote healing. We set the target on POD 3 for minor resection and POD 4 for major resection. $90 \%$ of patient $(n=9)$ in the minor group have achieved full diet by POD 3 . In the major group, similar to drains, 3 patients achieved full diet after POD4 due to ileus and prolonged intubation.

\section{CONCLUSION}

From these key targets and overall achievement in terms of complications, readmission, length of hospital stay, most of our targets have been met and comparable to other studies published previously. This shows that our ERAS protocol is feasible and safe to be applied to patient undergoing hepatectomy. We acknowledge that limitation in terms of small number of cases within this cohort. It was also noted that some patients had achieved their target but not discharged. The logistic and transportation issues, absence of close relatives to look after the patient and feeling afraid or not motivated for self-ambulation being the cause of delayed discharged days.

We advocate the use of this protocol as a checklist to help clinicians to avoid unnecessary prolonged hospital stay. This is a preliminary study for ERAS in liver resection and is a model to plan a proper prospective study with larger numbers in the future.

\section{ACKNOWLEDGEMENTS}

The authors thank all staff at the Department of Surgery and the Department of Statistics, Universiti Kebangsaan Malaysia.

Disclosure : the authors declare no conflict of interest.

\section{REFERENCES}

1. Kehlet H. Multimodal approach to control postoperative pathophysiology and rehabilitation. Br J Anaesth 1997; 78:606-617.

2. Masayuki Ishii, Toru Mizuguchi, Kohei Harada, Shigenori Ota, Makoto Meguro et al .
Comprehensive review of post-liver resection surgical complications and a new universal classification and grading system. World J Hepatol 2014; $27: 745-751$

3. Basse L, Hjort Jakobsen D, Billesbolle P, Werner M, Kehlet $\mathrm{H}$. A clinical pathway to accelerate recovery after colonic resection. Ann Surg 2000; 232:51-57.

4. Varadhan KK, Neal KR, Dejong CH, Fearon KC, Ljungqvist $\mathrm{O}$, Lobo DN. The enhanced recovery after surgery (ERAS) pathway for patients undergoing major elective open colorectal surgery: a meta-analysis of randomized controlled trials. Clin Nutr 2010; 29: 434-440.

5. Mackay G, O'Dwyer PJ. Early discharge following liver resection for colorectal metastases. Scott Med J 2008; 53:22-24.

6. Van Dam RM, Hendry PO, Coolsen MM, Bemelmans MH, Lassen K, Revhaug A et al.; Enhanced Recovery After Surgery (ERAS) Group. Initial experience with a multimodal enhanced recovery programme in patients undergoing liver resection. Br J Surg 2008; 95: 969-975.

7. Capussotti L, Muratore A, Ferrero A, Massucco $P$, Ribero D, Polastri R. Randomized clinical trial of liver resection with and without hepatic clamping. Br J Surg 2006; 93: 685-689

8. Jarnagin WR, Gonen M, Fong Y, DeMatteo RP, Ben-Porat L, Little $S$ et al. Improvement in perioperative outcome after hepatic resection. Analysis of 1803 consecutive cases over the past decade. Ann Surg 2002; 236: 397-406.

9. Jensen LS, Mortensen FV, Iversen MG, Jørgensen A, Kirkegaard P, Kehlet H. [Liver surgery in Denmark 2002-2007.] Ugeskr Laeger 2009; 171: 1365-1368.

10. Petrowsky H, McCormack L, Trujillo M et al. A prospective, randomized, controlled trial comparing intermittent portal triad clamping versus ischemic preconditioning with continuous clamping for major liver resection. Ann Surg 2006; 244: 921-928.

11. Block, B.M., S.S. Liu, A.J. Rowlingson, A.R. Cowan, J.A. Jr Cowan and C.L. Wu, Efficacy of postoperative epidural analgesia: a metaanalysis. JAMA 2003; 290(18): 2455-2463

12. Nelson, R., S. Edwards and B. Tse, . Prophylactic nasogastric decompression after abdominal surgery. Cochrane Database Syst Rev 2007; 3: CD004929.

13. Aldameh A1, MCCall JL, Koea JB. Is routine placement of surgical drains necessary after elective hepatectomy? Results from a single institution. J Gastrointest Surg. 2005;9(5):66771 